\title{
SÍNTESIS DE CATALIZADORES BASADOS EN Co-Mn PARA LA COMBUSTIÓN DE n-HEXANO
}

\author{
Gino Picasso ${ }^{a^{*}}$, José Quijano ${ }^{\mathrm{a}}$, Rosario Sun Kou ${ }^{\mathrm{b}}$
}

\begin{abstract}
RESUMEN
La combustión catalítica de n-hexano se ha estudiado utilizando diversos óxidos mixtos basados en Co-Mn por las propiedades rédox de estos materiales. Los catalizadores se prepararon mediante el método de co-precipitación usando un tiempo de envejecimiento 24 $\mathrm{h}$, partiendo de precursores a base de nitrato de $\mathrm{Co}^{2+} \mathrm{y} \mathrm{Mn}^{2+}$, en las proporciones molares $\mathrm{Co} /$ Mn de 5; 2; 1; 0,5 y 0,2. Los óxidos obtenidos fueron caracterizados por sorción de $\mathrm{N}_{2}$, por difracción de rayos $\mathrm{X}(\mathrm{XRD})$, por espectroscopía infrarroja de transformada de Fourier (FTIR) y por reducción a temperatura programada (TPR). Las isotermas de sorción de $\mathrm{N}_{2}$ indicaron que son materiales micro-mesoporososo con poros tipo cilíndricos. El análisis XRD reveló la presencia de fases cristalinas correspondientes a las especies mixtas tipo espinela $\mathrm{MnCo}_{2} \mathrm{O}_{4}$ y tetragonal, atribuido a $(\mathrm{CoMn})(\mathrm{CoMn})_{2} \mathrm{O}_{4}$. El análisis FTIR mostró señales desplazadas combinadas en las muestras mixtas, reflejando interacción metálica. El análisis TPR reveló mejor reducibilidad en las muestras mixtas comparado con los oxidos simples puros. Las curvas de ignición mostraron que el enriquecimiento de Co en la composición del catalizador mejoró la actividad, siendo el catalizador $\mathrm{Co} / \mathrm{Mn}=5$ el más activo, debido probablemente a la mejor superficie y mejor reducibilidad alcanzada.
\end{abstract}

Palabras clave: Combustión catalítica, n-hexano, Co-Mn.

\section{SYNTHESIS OF CATALYSTS BASED ON Co-Mn OXIDES FOR COMBUSTION OF n-HEXANE}

\begin{abstract}
Catalytic combustion of $\mathrm{n}$-hexane was studied over various mixed oxides based on Co-Mn by the redox properties of these materials. The catalysts were prepared by coprecipitation applying an aging time of $24 \mathrm{~h}$, from nitrate precursor of $\mathrm{Co}^{2+}$ and $\mathrm{Mn}^{2+}$ with molar $\mathrm{Co} / \mathrm{Mn}$ ratios corresponded to $5 ; 2 ; 1 ; 0,5$ and 0,2 . The oxides were characterized by $\mathrm{N}_{2}$ sorption (BET technique), X-ray diffraction (XRD), infrared Fourier transform spectroscopy (FTIR) and temperature programmed reduction (TPR). The isotherms of $\mathrm{N}_{2}$-sorption were of type IV corresponded to micro-mesoporous material, with the presence of cilindric pores. X-ray analysis revealed the presence of mixed phases corresponding to spinel $\mathrm{MnCo}_{2} \mathrm{O}_{4}$ and tetragonal $(\mathrm{CoMn})(\mathrm{CoMn})_{2} \mathrm{O}_{4}$. FTIR spectroscopy showed combined signals in the mixed samples, revealing metallic interaction. TPR analysis showed better reducibility in mixed samples in relation with the simple ones. The ignition curves for Co-Mn samples depicted better activities with the enrichment of Co compound in mixed samples, being the sample

\footnotetext{
a Laboratorio de Investigación de Fisicoquímica (LABINFIS). Facultad de Ciencias. Universidad Nacional de Ingeniería. Av. Túpac Amaru 210. Rímac. Lima-Perú *gpicasso@uni.edu.pe

${ }^{\mathrm{b}}$ Sección Química. Departamento de Ciencias. Pontifica Universidad Católica del Perú. Av. Universitaria 1801. San Miguel. Lima 32
} 
$\mathrm{Co} / \mathrm{Mn}=5$ the most efficiency, probably attributable to the best surface and the highest reducibility.

Keywords: Catalytic combustion, n-hexane, Co-Mn.

\section{INTRODUCCIÓN}

Los compuestos orgánicos volátiles ( $\mathrm{COV}^{\prime}$ s) son considerados como una clase importante de contaminantes del aire, que se definen según la Comisión Económica para Europa de las Naciones Unidas del año 1991 como "Compuestos orgánicos producidos por el hombre, distintos del metano, que son capaces de producir oxidantes fotoquímicos por reacción con óxidos de nitrógeno en presencia de luz solar". La oxidación catalítica es una de las más importantes y prometedoras opciones para la eliminación de los $\mathrm{COV}^{\prime} \mathrm{s}$, porque los procesos se realizan a temperaturas de operación menores de $500^{\circ} \mathrm{C}$, disminuyendo drásticamente la presencia de óxidos de nitrógeno generados por la contaminación térmica ${ }^{1}$.

El n-hexano es un líquido incoloro, volátil, inflamable $\left(\mathrm{T}_{\mathrm{eb}}=68,7^{\circ} \mathrm{C}\right)$, con olor característico, similar a la gasolina. Es un disolvente altamente inflamable muy usado en reacciones de polimerización y en la formulación de algunos productos adhesivos, lacas, cementos y pinturas. Es un componente importante de la gasolina de automóviles, muy utilizado como desnaturalizante de alcohol y en termómetros para temperaturas bajas en lugar de mercurio. Su presencia en la atmósfera está regulada por normas ambientales en la mayor parte de los países $^{2}$ y en el Perú, se realiza a través del Ministerio de Salud (MINSA) ${ }^{3}$. Por esta razón, el desarrollo de métodos para eliminar n-hexano es pertinente y necesario.

Los óxidos de manganeso presentan propiedades catalíticas atribuidas a la habilidad del $\mathrm{Mn}$ de formar óxidos dentro de un amplio rango de estructuras y fases cristalinas $\left(\beta-\mathrm{MnO}_{2}\right.$, $\gamma-\mathrm{MnO}_{2}, \alpha-\mathrm{MnO}_{2}, \gamma-\mathrm{Mn}_{2} \mathrm{O}_{3}, \alpha-\mathrm{Mn}_{2} \mathrm{O}_{3}$ y $\mathrm{Mn}_{5} \mathrm{O}_{8}$ ), donde los átomos de $\mathrm{Mn}$ se encuentran en diferentes estados de oxidación. Los óxidos de manganeso son también conocidos por su capacidad de almacenar oxígeno en su red cristalina y por la formación de estructuras sólidas amorfas, que presentan vacancias y promueve las reacciones de oxidación ${ }^{4}$. Se ha estudiado la oxidación del dióxido de manganeso para la combustión catalizada de metano, y se sugirió que una alta concentración de $\mathrm{Mn}^{3+}$ podría resultar en enlaces $\mathrm{Mn}-\mathrm{O}$ débiles, lo que podría asegurar una buena actividad catalítica ${ }^{5}$. Catalizadores basados en otras especies de óxidos de $\mathrm{Mn}$ soportados en zirconia, como $\mathrm{Mn}_{2} \mathrm{O}_{3}$, han sido aplicados para remover monóxido de carbono y óxidos de nitrógeno de los tubos de escape de autos ${ }^{5}$ y como agente almacenador de oxígeno ${ }^{6,7}$. El óxido $\alpha-\mathrm{Mn}_{2} \mathrm{O}_{3}$ también ha sido usado como catalizador y combustor ya que puede absorber oxígeno y actuar en una atmósfera oxidante, debido a la posibilidad del catión $\mathrm{Mn}^{+3}$ de enlazarse formado diferentes centros activos ${ }^{7,8}$.

Por otro lado, los óxidos de cobalto se presentan como los materiales más prometedores en la remoción de $\mathrm{CO}$ debido a la presencia de oxígeno móvil en la estructura de la espinela $\mathrm{Co}_{3} \mathrm{O}_{4}{ }_{4}$. La alta actividad de este óxido en la oxidación de $\mathrm{CO}$ es atribuida a la débil fuerza en el enlace $\mathrm{Co}-\mathrm{O}$, la cual crea una fácil interacción entre los oxígenos de la red cristalina con los reactantes ${ }^{9,10}$. Entre las especies de óxidos de cobalto, se reportó que el $\mathrm{Co}_{3} \mathrm{O}_{4}$ es un buen 
candidato para la combustión catalítica, evaluándose su actividad para diferentes $\mathrm{COV}^{\prime} \mathrm{s}^{11}$. Al comparar los trabajos realizados con óxidos de cobalto en diferentes soportes se encontró que los catalizadores de $\mathrm{Co}_{3} \mathrm{O}_{4}$ soportados en $\mathrm{ZrO}_{2}{ }^{12}$, tienen mayor actividad en comparación con los catalizadores de $\mathrm{Co}_{3} \mathrm{O}_{4}$ soportados en $\mathrm{Al}_{2} \mathrm{O}_{3}{ }^{13}$. Se estudiaron, además, sistemas $\mathrm{Co}_{3} \mathrm{O}_{4} /$ $\mathrm{CeO}_{2}$ y $\mathrm{Co}_{3} \mathrm{O}_{4} / \mathrm{CeO}_{2}-\mathrm{ZrO}_{2}$ con un $30 \%$ (en peso) de la espinela, encontrándose que la ceria y la ceria-zirconia ayudan a dispersar la fase activa sobre el soporte y promueven la oxidación a baja temperatura ${ }^{14-15}$. La literatura señala que los óxidos tipo $\mathrm{Co}_{3} \mathrm{O}_{4}$ constituyen los mejores catalizadores para la oxidación catalítica de tolueno y de propeno ${ }^{16}$.

También se ha reportado que las estructuras tipo hidrotalcitas, preparadas por la modificación de la composición química de los óxidos, tanto de manganeso como de cobalto con cobre, han presentado mejores actividades respecto a los óxidos simples, para la combustión catalítica de $\mathrm{COV}^{\prime} \mathrm{s}^{17}$. Adicionalmente, los complejos metálicos del tipo $\mathrm{Cu} / \mathrm{CoMgAl}$ y $\mathrm{Mn}-\mathrm{Co}-\mathrm{Al}$ han mostrado buena actividad catalítica para la combustión completa de etanol y tolueno ${ }^{18,19}$ y en forma similar los sistemas tipo Co-O-Mn, tuvieron un buen comportamiento catalítico en la combustión completa de tolueno ${ }^{20}$.

En este trabajo se preparó catalizadores basados en óxidos mixtos de Co-Mn por coprecipitación, empleando bicarbonato de sodio y nitrato de amonio como agentes precipitantes, con un tiempo de envejecimiento de $24 \mathrm{~h}$. El objeto de este trabajo fue determinar la influencia del efecto sinérgico de los componentes del catalizador en las propiedades catalíticas, así como de las variables experimentales de la síntesis, en la formación de la fase activa de los catalizadores, en su estructura cristalina y características texturales, para poder obtener un sistema mixto activo, selectivo y estable en la reacción de combustión completa de n-hexano.

\section{Materiales de partida}

\section{PARTE EXPERIMENTAL}

Los reactivos de partida usados para la preparación de los catalizadores basados en óxidos mixtos de Co-Mn, se detallan en la tabla $1 .$.

Tabla 1. Reactivos de partida para la preparación de los catalizadores mixtos de CoMn.

\begin{tabular}{ccc}
\hline Medio de solución & Agente precipitante & Sales metálicas \\
\hline \multirow{3}{*}{ Agua destilada } & Bicarbonato de sodio, $\mathrm{NaHCO}_{3}$ & Nitrato de cobalto hexahidratado \\
& (MERCK) & $\mathrm{Co}\left[\left(\mathrm{NO}_{3}\right)\right]_{2} \cdot 6 \mathrm{H}_{2} \mathrm{O}(\mathrm{ALDRICH})$. \\
\cline { 2 - 3 } & Nitrato de amonio, $\mathrm{NH}_{4} \mathrm{NO}_{3}$ & Nitrato de manganeso (II) tetrahidratado \\
& $(\mathrm{MERCK})$ & $\mathrm{Mn}\left[\left(\mathrm{NO}_{3}\right)\right]_{2} .4 \mathrm{H}_{2} \mathrm{O}$ (MERCK) \\
\hline
\end{tabular}




\section{Preparación de los catalizadores}

Se preparó catalizadores basados en óxidos mixtos de Co-Mn mediante el método de coprecipitación a partir de soluciones molares de $0,10 \mathrm{M}$ a base de sales de $\mathrm{Co}^{2+} \mathrm{y} \mathrm{Mn}^{2+}$ (ecuación 1), mezclados en cantidades predeterminadas para obtener las muestras mixtas de $\mathrm{Co}_{\mathrm{x}} \mathrm{Mn}_{\mathrm{y}}$ con las relaciones molares $\mathrm{Co} / \mathrm{Mn}$ correspondientes a $5 ; 2 ; 1 ; 0,5$ y 0,2 . Las soluciones de las sales metálicas se precipitaron con ayuda de soluciones $0,1 \mathrm{M}$ de $\mathrm{NaHCO}_{3} \mathrm{y} \mathrm{NH}_{4} \mathrm{NO}_{3}{ }^{18-20}$ (ecuación 2). La solución metálica mixta preparada se agitó, teniendo una primera serie agitando durante $4 \mathrm{~h}$ y otra durante 1 hora a un $\mathrm{pH}$ entre 7 a 8,5 , correspondiente a la precipitación total para los diferentes óxidos, usando un $\mathrm{pHmetro}$ digital con un electrodo de referencia de plata/cloruro de plata $(\mathrm{Ag} / \mathrm{AgCl})$. Se dejó envejecer durante 24h, luego se filtró al vacío los polvos obtenidos (bomba de vacío BODECO Germany R-300), enjuagándolos con $100 \mathrm{~mL}$ de agua destilada por 4 veces para remover los restos de iones sodio $\mathrm{Na}^{+}$que puedan quedar en el precipitado. Luego, el sólido remanente se enjuagó con etanol para eliminar algún compuesto orgánico presente en la superficie, se secó a $25^{\circ} \mathrm{C}$ durante $24 \mathrm{~h}$ en ambiente deshidrantante. Finalmente, se calcinó en aire en un horno TERMOLINE TZ4ST, precalentado inicialmente a $250^{\circ} \mathrm{C}$ hasta la temperatura de $550^{\circ} \mathrm{C}$ durante $4 \mathrm{~h}$, empleando una rampa de calentamiento de $10^{\circ} \mathrm{C} / \mathrm{min}$. Las reacciones químicas correspondientes fueron las siguientes:

$$
\begin{aligned}
& \mathrm{Co}\left(\mathrm{NO}_{3}\right)_{2} \cdot 6 \mathrm{H}_{2} \mathrm{O}+\mathrm{Mn}\left(\mathrm{NO}_{3}\right)_{2} \cdot 4 \mathrm{H}_{2} \mathrm{O}+\mathrm{H}_{2} \mathrm{O}_{(\mathrm{l})} \rightarrow \mathrm{Co}^{2+}+\mathrm{Mn}^{2+}+4 \mathrm{NO}_{3}^{-}+11 \mathrm{H}_{2} \mathrm{O}_{(\mathrm{l})} \quad \text { Ec. } 1 \\
& \mathrm{xCo}^{2+}+\mathrm{yMn}^{2+}+2 \mathrm{NH}_{4} \mathrm{NO}_{3}+2 \mathrm{NaHCO}_{3} \rightarrow \downarrow \mathrm{Co}_{\mathrm{x}} \mathrm{Mn}_{\mathrm{y}}+2 \mathrm{CO}_{3}{ }^{2-}+2 \mathrm{Na}^{+}+2 \mathrm{NH}_{4}^{+}+2 \mathrm{H}^{+}+2 \mathrm{NO}_{3}^{-} \text {Ec. } 2
\end{aligned}
$$

Los óxidos obtenidos fueron representados como $\mathrm{Co}_{x} \mathrm{Mn}_{y}$, donde " $\mathrm{x}$ " e " $\mathrm{y}$ " representan la composición molar correspondiente al cobalto y al manganeso, respectivamente. Los métodos de caracterización empleados con las muestras fueron los siguientes:

\section{Análisis termogravimétrico (TGA)}

Se aplicó la técnica TGA para el registro de manera continua de la pérdida de masa del carbonato producido en la ecuación (2), en función de la temperatura; el análisis se realizó en atmósfera controlada de nitrógeno. Se utilizó un equipo SDT Q600 V 20,9, utilizando una masa inicial de muestra de 25 a $50 \mathrm{mg}$, con una rampa de temperatura de $20^{\circ} \mathrm{C}$ hasta $800^{\circ} \mathrm{C}$ y con una velocidad de calentamiento de $5^{\circ} \mathrm{C} / \mathrm{min}$.

\section{Sorción de $\mathrm{N}_{2}$ (método $\mathrm{BET}$ )}

La técnica de sorción de $\mathrm{N}_{2}$ sirve para la medición de la superficie específica; para este ensayo se utilizó un equipo Micromeritics GEMINI-VII serie t, ubicado en el Laboratorio de Investigación de Fisicoquímica de la Facultad de Ciencias (LABINFIS-UNI). Se utilizó $\mathrm{N}_{2}$ como gas de análisis $\left(\mathrm{N}_{2}, 99 \%\right)$ y nitrógeno líquido con punto de ebullición a $77 \mathrm{~K}$ como refrigerante. Los puntos de equilibrio se obtuvieron siguiendo el programa GAS MICROMERITICS con un total de 30 a 50 puntos y con un tiempo de equilibrio de $6 \mathrm{~s}$ en cada punto. Previo a la adsorción, las muestras fueron desgasificadas en ambiente inerte durante $2 \mathrm{~h}$ a $250{ }^{\circ} \mathrm{C}$. 


\section{Espectroscopía infrarroja con transformada de Fourier (FTIR)}

La técnica FTIR se usó para la identificación de los grupos funcionales y modos de vibración presentes en las muestras preparadas en forma de pastilla con $\mathrm{KBr}$. Se utilizó un espectrómetro FTIR marca IR Prestige21 Shimadzu, ubicado en la Facultad de Ciencias (UNI), cuyo espectro se obtuvo con el programa $\mathrm{ACD} / \mathrm{SpecViewer}$, registrando los picos característicos de enlaces que presenten resonancia en un amplio rango de longitud de onda entre los 4000 a $400 \mathrm{~cm}^{-1}$ y con una resolución de $4 \mathrm{~cm}^{-1}$.

\section{Difracción de rayos $\mathrm{X}$ (XRD)}

La técnica XRD se utilizó para la identificación cristalográfica de las fases cristalinas presentes en las muestras. El análisis XRD se realizó en un equipo RIGAKU Modelo Miniflex, ubicado en el IPEN, con tubo de $\mathrm{Cu}$ y filtro de $\mathrm{Ni}$, con $30 \mathrm{kV}$ y $15 \mathrm{~mA}$; se usó un monocromador de grafito con el objeto de seleccionar la radiación $\mathrm{CuK} \alpha$ del ánodo de cobre, y se ha trabajado con ángulos de barrido entre 5 y $100^{\circ}$ con una velocidad del goniómetro de 0,04 \% .

\section{Análisis de temperatura programada de reducción (TPR)}

Los experimentos de reducción a temperatura programada (TPR) se realizaron en un equipo Micromeritics CHEMISORB 2720, ubicado en LABINFIS-UNI. Las medidas se realizaron con las muestras pretratadas a $200{ }^{\circ} \mathrm{C}$ por $2 \mathrm{~h}$ en ambiente inerte para extraer el agua e impurezas y luego calentando las muestras desde 25 hasta los $900{ }^{\circ} \mathrm{C}$ en atmósfera reductora $\left(10 \%\right.$ de $\mathrm{H}_{2}$ en $\left.\mathrm{N}_{2}\right)$ y con una rampa de calentamiento de $10^{\circ} \mathrm{C}$ por minuto. Para la calibración del instrumento se usó una muestra de $\mathrm{Ag}_{2} \mathrm{O}$ estandarizada, que presenta un pico característico de consumo de $\mathrm{H}_{2}$ a $140{ }^{\circ} \mathrm{C}$.

\section{Medición de la actividad catalítica}

La actividad de los óxidos mixtos se evaluó en un reactor de lecho fijo, consistente en un tubo de cuarzo con frita del mismo material donde se colocó $100 \mathrm{mg}$ de catalizador mezclado con $200 \mathrm{mg}$ de cuarzo, para mitigar los puntos calientes. La corriente de alimentación al reactor se obtuvo con una serie de saturadores a $0^{\circ} \mathrm{C}$ para obtener una concentración inicial de n-hexano de $2000 \mathrm{ppm}$ con una velocidad espacial respecto a la masa de $80 \mathrm{~h}^{-1}$ y un flujo de alimentación de $100 \mathrm{~mL} / \mathrm{min}$. Los flujos de gas en régimen estacionario se fijó en el sistema de reacción mediante reguladores másicos ALICAT, previamente calibrados, que registran los flujos digitalmente. Los productos de la reacción catalítica se analizaron con el detector FID del cromatógrafo de gases VARIAN GC-450, usando una columna empacada CARBOWAX. Las curvas de ignición se obtuvieron graficando la relación entre la temperatura de combustión y el porcentaje de conversión de n-hexano a $\mathrm{CO}_{2} \mathrm{y}_{2} \mathrm{O}$. En este trabajo no se observó productos de combustión incompleta. El esquema detallado del equipo se ha mostrado en un trabajo previo ${ }^{21}$.

\section{RESULTADOS Y DISCUSIÓN}

Los análisis termogravimétricos se realizaron en atmósfera inerte de $\mathrm{N}_{2}$, con el objetivo de determinar la temperatura de descomposición de los carbonatos y su transformación en óxidos (Ec.3). Los termogramas TGA de las muestras con un tiempo de envejecimiento de 
24h se presentan en la figura 1a.

$$
\mathrm{MCO}_{3} \underset{\Delta}{\longrightarrow} \mathrm{M}_{\mathrm{X}} \mathrm{O}_{\mathrm{Y}}+\mathrm{CO}_{2} \quad \text { Ec. } 3
$$

Como se observa en la figura 1a, la temperatura de descomposición de los carbonatos se encuentra entre 300 a $400^{\circ} \mathrm{C}$. Por esta razón, la temperatura de calcinación fijada para todas las muestras fue de $550{ }^{\circ} \mathrm{C}$, considerando que a partir de esta temperatura no se observó pérdida de masa en ninguna de las muestras. Como ejemplo representativo, el porcentaje de pérdida de masa, así como las reacciones de descomposición del óxido mixto Co1Mn2 con las correspondientes a los óxidos puros $\mathrm{CoO}_{\mathrm{x}}$ y $\mathrm{MnO}_{\mathrm{x}}$ se presentan en la tabla 2. Se observa que los porcentajes de pérdidas de masa teóricos coinciden, en general, con los experimentales.

En la tabla 3 se presentan los resultados de las superficies específicas de los catalizadores basados en óxidos mixtos $\mathrm{Co}_{\mathrm{x}} \mathrm{Mn}_{\mathrm{y}}$ con un tiempo de envejecimiento de 24h. En todas las muestras, las isotermas de sorción de $\mathrm{N}_{2}$ son del tipo IV correspondientes a materiales micro-mesoporosos con mayor presencia de poros cilíndricos (Figura 1b). Como se observa de la tabla 3, existe una tendencia a incrementarse la superficie específica con el aumento del contenido molar de cobalto, llegando a un valor máximo de $116 \mathrm{~m}^{2} / \mathrm{g}$ para la muestra Co5Mn1 en comparación con las muestras Co1Mn1 y Co2Mn1 que presentan una superficie específica de 46 y $69 \mathrm{~m}^{2} / \mathrm{g}$, respectivamnente. Además, con estos catalizadores (Co1Mn1, Co2Mn1, Co5Mn1) se observa que con el incremento del contenido de Co se produce una disminución de tamaño de poro debido posiblemente a la formación de especies de Co más pequeñas (tabla 3 ).
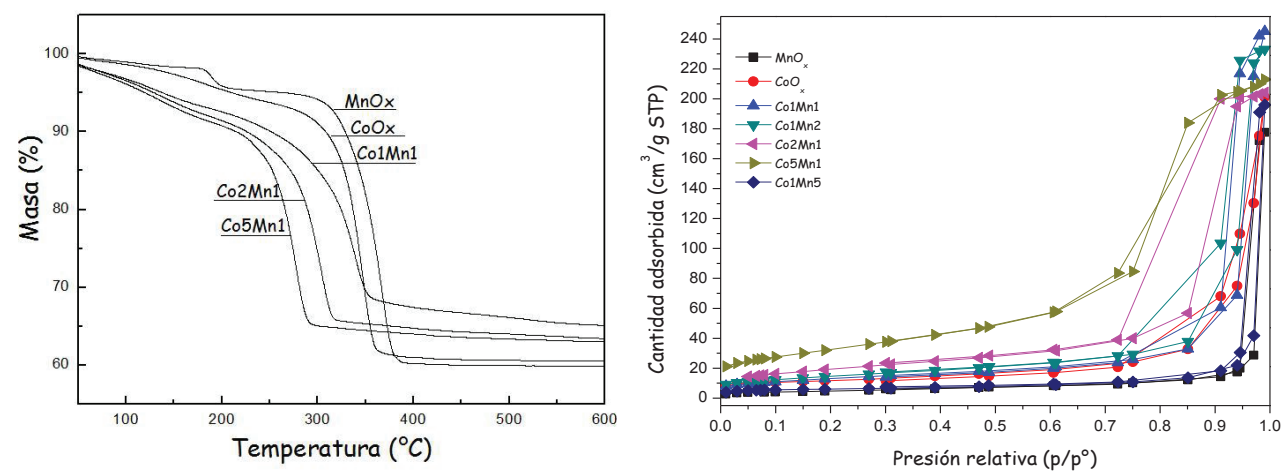

Figura 1. Termogramas TGA (a) e isotermas de sorción de $\mathrm{N}_{2}$ (b) de las muestras $\mathrm{Co}_{\mathrm{x}} \mathrm{Mn}_{\mathrm{y}}$ con un tiempo de envejecimiento de $24 \mathrm{~h}$. 
Tabla 2. Reacciones de descomposición y \% de pérdida de masa de los óxidos mixtos y simples con tiempo de envejecimiento de $24 \mathrm{~h}$

\begin{tabular}{ccccc}
\hline Código & $\begin{array}{c}\text { Fórmula del } \\
\text { Carbonato }\end{array}$ & Reacción de descomposición & $\begin{array}{c}\text { \% de pérdida } \\
\text { de masa } \\
\text { teórico }\end{array}$ & $\begin{array}{c}\text { \% de pérdida } \\
\text { de masa } \\
\text { experimental }\end{array}$ \\
\hline $\mathrm{CoO}_{\mathrm{x}}$ & $\mathrm{CoCO}_{3}$ & $\mathrm{CoCO}_{3(\mathrm{~s})} \overrightarrow{300^{\circ} \mathrm{C}} \mathrm{CoO}_{(s)}+\mathrm{CO}_{2(\mathrm{~g})} \uparrow$ & 63,01 & 62,74 \\
$\mathrm{MnO}_{\mathrm{x}}$ & $\mathrm{MnCO}_{3}$ & $\mathrm{MnCO}_{3(\mathrm{~s})} \overrightarrow{400^{\circ} \mathrm{C}} \mathrm{MnO}_{(s)}+\mathrm{CO}_{2(\mathrm{~g})} \uparrow$ & 61,72 & 59,52 \\
$\mathrm{Co}_{\mathrm{Mn} 2}$ & $\begin{array}{l}\mathrm{CoCO}_{3}+ \\
2 \mathrm{MnCO}_{3}\end{array}$ & $\mathrm{CoCO}_{3(\mathrm{~s})}+2 \mathrm{MnCO}_{3(\mathrm{~s})} \overrightarrow{375^{\circ} \mathrm{C} \mathrm{CoO}_{(s)}+2 \mathrm{MnO}_{(s)}+3 \mathrm{CO}_{2(\mathrm{~g})} \uparrow}$ & 62,16 & 60,23 \\
\hline
\end{tabular}

Tabla 3. Parámetros texturales de los diferentes óxidos con tiempo de envejecimiento de $24 \mathrm{~h}$.

\begin{tabular}{|c|c|c|c|c|c|c|}
\hline \multirow{2}{*}{$\begin{array}{l}\text { Óxidos } \\
\text { Mixtos }\end{array}$} & \multirow{2}{*}{$\begin{array}{l}\text { Relación } \\
\text { molar } \\
\text { (Co/Mn) }\end{array}$} & \multirow{2}{*}{$\begin{array}{c}\text { Area } \\
\text { superficial } \\
\text { BET }\left(\mathrm{m}^{2} / \mathrm{g}\right)\end{array}$} & \multirow{2}{*}{$\begin{array}{l}\text { Volumen de } \\
\text { poro (Método } \\
\text { t- plot) }\left(\mathrm{cm}^{3} / \mathrm{g}\right)\end{array}$} & \multicolumn{2}{|c|}{$\begin{array}{c}\text { Tamaño de poro } \\
(\text { Método BJH) }(\mathrm{nm})\end{array}$} & \multirow{2}{*}{$\begin{array}{c}\text { Tamaño de } \\
\text { cristalita }(\mathrm{nm})\end{array}$} \\
\hline & & & & $\begin{array}{c}\text { BJH } \\
\text { Adsorción }\end{array}$ & $\begin{array}{c}\text { BJH } \\
\text { Desorción }\end{array}$ & \\
\hline $\mathrm{CoOx}$ & Óxido de Co & 40 & 0,0192 & 13,6 & 8,6 & 30,9 \\
\hline MnOx & Óxido de Mn & 17 & 0,0024 & 27,9 & 21,5 & - \\
\hline Co1Mn1 & 1 & 46 & 0,0068 & 14,6 & 10,5 & 13,7 \\
\hline Co1Mn2 & 0,5 & 32 & 0,0125 & 12,2 & 7,2 & 17,4 \\
\hline Co2Mn1 & 1 & 69 & 0,0486 & 7,5 & 4,0 & 10,3 \\
\hline Co5Mn1 & 5 & 116 & 0,5258 & 4,4 & 4,0 & 6,9 \\
\hline Co1Mn5 & 0,2 & 20 & 0,0020 & 31,7 & 21,2 & - \\
\hline
\end{tabular}

Los difractogramas XRD de las muestras $\mathrm{Co}_{\mathrm{x}} \mathrm{Mn}_{\mathrm{y}}$ se presentan en la figura 3a. El XRD correspondiente al óxido puro de cobalto $\mathrm{CoO}_{x}$ presenta los picos caracteristicos a $2 \Theta=$ 31,$37 ; 36,88 ; 44,87 ; 59,39$ y 65,29 que corresponden con la formación de la espinela $\mathrm{Co}_{3} \mathrm{O}_{4}$ (JCPDS card $\mathrm{n}^{\circ}$. 43-1003), con ausencia de la fase CoO. Los picos de difracción de las muestras Co5Mn1, Co2Mn1, Co1Mn1, Co1Mn2, Co1Mn5 presentan un corrimiento hacia menores ángulos a medida que aumenta la composición molar de Mn en la estructura, con la consiguiente expansión del parámetro de red de la celda unitaria debido posiblemente al mayor tamaño de las especies $\mathrm{Mn}^{+2}(\mathrm{r}=0,080 \mathrm{pm})$ y $\mathrm{Mn}^{+3}(\mathrm{r}=0,066 \mathrm{pm})$ frente a las especies más pequeñas $\mathrm{Co}^{+2}(\mathrm{r}=0,072 \mathrm{pm})$ y $\mathrm{Co}^{+3}(\mathrm{r}=0,063 \mathrm{pm})$, formando una estructura espinela poco distorsionada ${ }^{22}$. En las muestras Co1Mn1, Co2Mn1, Co5Mn1, la distorsión se puede verificar por la disminución del tamaño de la cristalita (figura $3 \mathrm{~b}$ ) con el aumento del contenido de $\mathrm{Co}$, a diferencia de las muestras Co1Mn2, Co1Mn5, ricas en Mn, que presentaron una cristalinidad similar a la espinela $\mathrm{Co}_{3} \mathrm{O}_{4}$. Se observó una buena correlación de la reducción de la superficie específica con el tamaño de cristalita (figura $3 \mathrm{~b}$ ). Otra carácterística importante es el aumento de la superficie específica del sistema $\mathrm{Co}_{\mathrm{x}} \mathrm{Mn}_{\mathrm{y}}$ respecto al correspondiente a $\mathrm{MnOx}$, que es poco cristalino (tabla 3, fig.2). Las distorsiones observadas en los difractogramas de las muestras CoxMny respecto a la espinela $\mathrm{Co}_{3} \mathrm{O}_{4}$ son un indicativo de la ausencia de una 
segregación de las fases, lo que permite poder descartar la formación de una mezcla simple de óxidos individuales.

El XRD correspondiente a la muestra $\mathrm{MnO}_{\mathrm{x}}$ presenta al óxido de manganeso puro con los picos de difracción a $2 \Theta=28,9 ; 33,5 ; 37,3$ y 56,8 que corresponden a la fase pirolusita $\beta-\mathrm{MnO}_{2}$ (PDF-00-004-0779, pirolusita), a la fase tipo hausmanita $\mathrm{Mn}_{3} \mathrm{O}_{4}$ (JCPDS $\mathrm{N}^{\circ} 24-$ 0734), las señales $2 \Theta=18,5 ; 35,7$ y 58,1 también son atribuidas a la fase $\mathrm{Mn}_{5} \mathrm{O}_{8}$ (JCPDS $\mathrm{N}^{\circ} 39$ a 1218$)^{23}$, pero estas señales se solapan en las otras especies de óxido de manganeso.
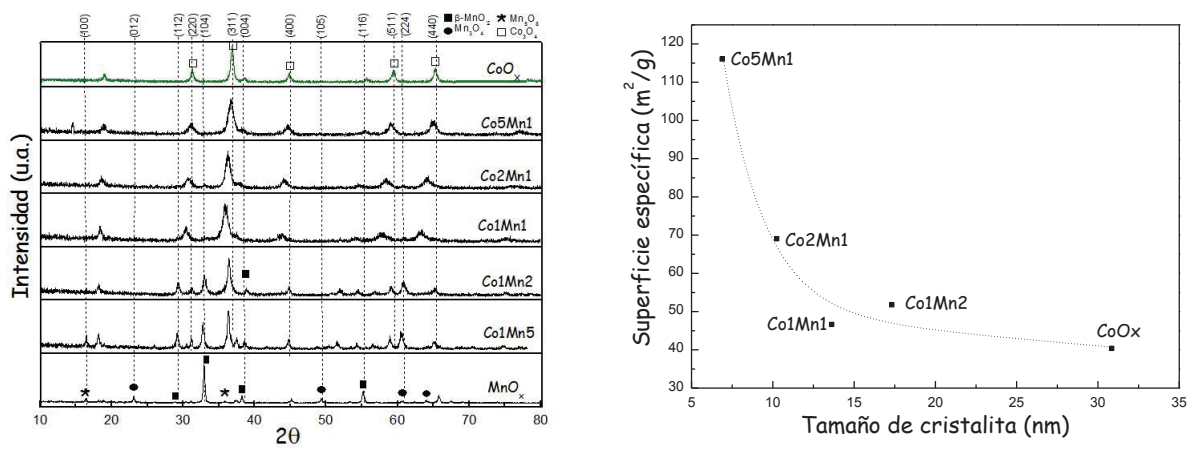

Figura 2. Difractogramas XRD (a) y correlación de la magnitud de la superficie específica con el tamaño de cristalita (b) de los óxidos mixtos de CoxMny y óxidos simples $\mathrm{CoOx}$ y $\mathrm{MnOx}$.

Los espectros FTIR de los óxidos $\mathrm{Co}_{\mathrm{x}} \mathrm{Mn}_{\mathrm{y}}$ se muestran en la figura $3 \mathrm{a}$, en donde se observa un pico ancho aproximadamente a $3500 \mathrm{~cm}^{-1}$, que se atribuiye a los modos vibracionales de

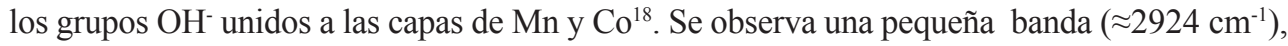
que se debe a la unión del hidrógeno del agua con los aniones carbonatos remantes $\mathrm{CO}_{3}{ }^{2-}$. Los picos entre 1423 y $1442 \mathrm{~cm}^{-1}$ se pueden atribuir a la vibración antisimétrica de $\mathrm{CO}_{3}^{2-}$. Las bandas de $\mathrm{CO}$ se desplazan para reducir los números de onda cuando el cobalto sustituye progresivamente al $\mathrm{Mn}$.

La banda ancha alrededor de los $875 \mathrm{~cm}^{-1}$ en $\mathrm{Co} 1 \mathrm{Mn} 2, \mathrm{CoO}_{\mathrm{x}}$ y $\mathrm{MnO}_{\mathrm{x}}$, es asignada al ángulo de deformación $\mathrm{H}-\mathrm{O}-\mathrm{H}$ de las moléculas de agua formadas en la capa intermedia de los óxidos.

Los picos observados en la región entre $550-600 \mathrm{~cm}^{-1}$ se atribuye a la vibración tensional $\mathrm{Co}^{+3}-\mathrm{O}$ y en la región $650-700 \mathrm{~cm}^{-1}$, a la vibración tensional $\mathrm{Co}^{+2}-\mathrm{O}$. El ligero corrimiento de los espectros FTIR en las muestras mixtas respecto a la muestra $\mathrm{CoOx}$ hacia menores frecuencias se puede atribuir a una disminución en la fuerza del enlace $\mathrm{Co}-\mathrm{O}$ en $\mathrm{Co}_{3} \mathrm{O}_{4}$, que es otro indicativo de la presencia de especies mixtas $\mathrm{Co}-\mathrm{Mn}^{24}$.

Los termogramas TPR correspondientes a los óxidos mixtos se diferencian sustancialmente de su contraparte atribuidos a los óxidos puros (figura 3b). El perfil TPR de MnOx muestra 
una banda ancha en lugar de picos diferenciados que puede ser debido a la reducción del $\mathrm{MnO}_{2}$ en varios estadios de oxidación ${ }^{21}$. En el caso del CoOx, el TPR muestra la presencia de 2 picos: uno pequeño, a $261^{\circ} \mathrm{C}$ y otro bastante ancho, a $435^{\circ} \mathrm{C}$, correspondiente a la reducción del $\mathrm{Co}^{+3} \mathrm{a} \mathrm{Co}^{+2} \mathrm{y}$ del $\mathrm{Co}^{+2} \mathrm{a} \mathrm{Co}$, respectivamente.

Los TPR de los óxidos mixtos presentan 2 picos, como en la muestra Co1Mn1 que se desplazan a menores temperaturas a medida que aumenta la composición del Co en la muestra mixta, ello indicaría que con el aumento de Co se mejora el grado de reducibilidad de los óxidos mixtos. La evolución en los termogramas se podría atribuir a la reducción de las diferentes especies del sistema mixto, y no con la reducción de una segregación de óxidos puros, confirmando el análisis XRD discutido anteriormente.

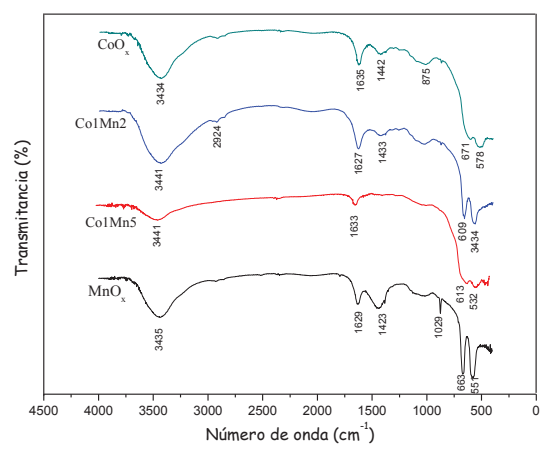

a

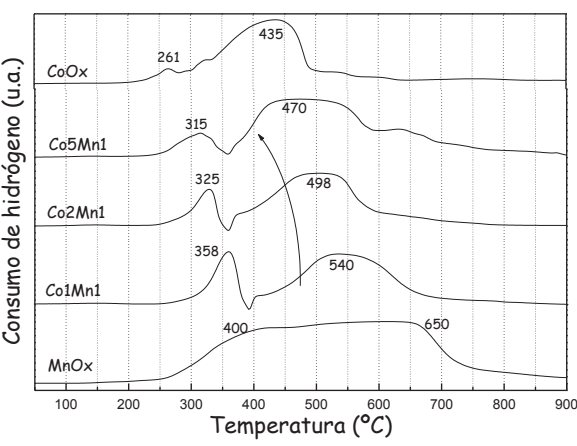

b

Figura 3. Espectros FTIR (a) y termogramas TPR (b) de los óxidos mixtos $\mathrm{Co}_{x} \mathrm{Mn}_{y} \mathrm{y}$ de los óxidos puros.

\section{Actividad catalítica}

En la figura 4a se presenta las curvas de ignición en la combustión de n-hexano obtenidas con los óxidos mixtos de Co-Mn con un tiempo de envejecimiento de $24 \mathrm{~h}$. Se observa una mayor actividad en los catalizadores con un mayor contenido de Co en la estructura del óxido mixto, debido posiblemente a un aumento de la superficie específica, por ejemplo en las muestras $\mathrm{MnOx}, \mathrm{Co} 1 \mathrm{Mn} 1, \mathrm{Co} 2 \mathrm{Mn}, \mathrm{Co} 5 \mathrm{Mn} 1$, las superficies son 17, 46, 69 y 116, respectivamente (tabla 3). Adicionalmente, comparando los TPR del MnOx puro con el Co5Mn1 se observa una mejora en el nivel de reducibilidad (figura 3b) que facilita la participación del oxígeno de red del catalizador promocionando la combustión completa del n-hexano. En referencia al CoOx puro, a pesar que la superficie del mismo es mucho menor que la correspondiente al Co5Mn1 (40 vs $116 \mathrm{~m}^{2} / \mathrm{g}$ ), las temperaturas de combustión completa del n-hexano son similares (figura 4b). En este caso, el factor controlante no parece ser la superficie sino la conformación estructural de la espinela formada y la facilidad de transferir oxígeno estructural, que se verificó por las menores temperaturas de reducción de la muestra $\mathrm{CoOx}$ respecto a la muestra $\mathrm{Co}_{5} \mathrm{Mn} 1$ (figura $3 \mathrm{~b}$ ). Adicionalmente, un aspecto importante, como apuntan algunos autores ${ }^{23,24}$, es el método de preparación que favoreció la formación de 
especies finamente divididas de $\mathrm{CoOx}$ en gran proporción con la formación simultánea de especies $\mathrm{Co}^{+2} \mathrm{y} \mathrm{Co}^{+3}$ y el enriquecimiento en especies de oxígeno superficial, factores que han contribuido a un aumento en la capacidad de reducibilidad de estas muestras.

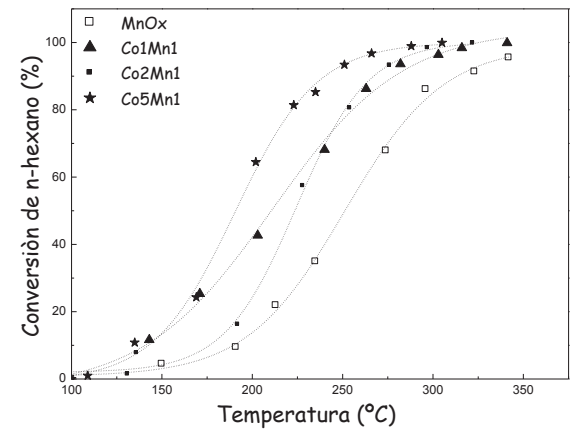

a

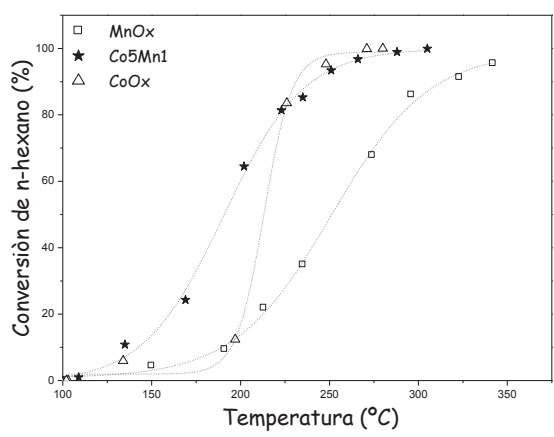

b

Figura 4. Curvas de conversión de n-hexano de los óxidos mixtos $\mathrm{Co}_{\mathrm{x}} \mathrm{Mn}_{\mathrm{y}}$ (a) y comparación de la curva de la muestra Co5Mn1 con la correspondiente a las muestras simples (b).

\section{CONCLUSIONES}

1. Se han prepararon óxidos mixtos basados en Co-Mn por el método de co-precipitación con tiempo envejecimiento de $24 \mathrm{~h}$, que ha permitido obtener áreas superficiales BET mayores conforme la proporción molar de Co aumenta en la composicón del óxido. El análisis TGA permitió obtener la temperatura a la cual los carbonatos se descomponen, siendo esta temperatura alrededor de $\operatorname{los} 400^{\circ} \mathrm{C}$.

2. El espectro FTIR de los óxidos mixtos mostró picos caracteristicos en la región entre $550-700 \mathrm{~cm}^{-1}$, que representan los modos de vibración de los enlaces $\mathrm{Co}^{+3}-\mathrm{O}$ y $\mathrm{Co}^{+2}-\mathrm{O}$ en posiciones desplazadas respecto a los espectros FTIR del óxido puro CoOx. Los difratogramas XRD revelaron la formación de una estructura tipo espinela para el óxido de cobalto puro y una estructura espinela poco distorsionada en los óxidos mixtos, lo que al parecer ha sido adecuada para mejorar su actividad catalítica. El análisis TPR de las muestras mixtas mostró una tendencia al aumento en la reducibilidad con el aumento de la composición molar del Co, confirmando la presencia de especies mixtas en diferentes estados de oxidación.

3. Las curvas de ignición mostraron una mayor actividad en los catalizadores con mayor presencia del Co en la composición molar del óxido mixto debido probablemente al aumento de la superficie específica y de la capacidad reductora obtenida. 


\section{AGRADECIMIENTO}

Los autores agradecen al Instituto de Investigación (IGI-UNI) y a la Facultad de Ciencias de la Universidad Nacional de Ingeniería por la subvención económica recibida para la realización de este trabajo.

\section{BIBLIOGRAFÍA}

1. Everaert K., Baeyens J., Catalytic combustion of volatile organic compounds, J. Hazard. Mater. 2004; 109 (1-3): 113-139.

2. Norma Oficial Mexicana NOM-047-SSA1-2011, Salud ambiental- Indices biológicos de exposición para el personal ocupacionalmente expuestos a sustancias químicas.

3. MINSA, Decreto Ministerial No 07-048071-002 que contiene los límites permisibles y efectos sobre la salud de sustancias químicas (n- hexano).

4. Kim S., Shim S., Catalytic combustion of VOC's over a series of manganese oxide catalysts, Appl. Catal. B 2010; 98: 180-185.

5. Zhang H. M., Teraoka Y., Yamazoe H., Effects of preparation methods on the methane combustion activity of supported Mn2O3 and LaMn-O3 catalysts, Catal. Today. 1989; 6, 155-162.

6. Craciun R., Nentwick B., Hadjiivanov K., Knozinger H., Structure and redox properties of $\mathrm{MnOx} /$ Yttrium-stabilized zirconia (YSZ) catalyst and its used in $\mathrm{CO}$ and $\mathrm{CH} 4$ oxidation, Appl. Catal. A 2003; 243: 67-79

7. Chang Y.F., McCarty J.G., Novel oxygen storage components for advanced catalysts for emission control in natural gas fueled vehicles, Catal.Today 1996; 30, 163-170.

8. Lamaita L., Peluso M., Sambeth J., Thomas H., Mineli G., Porta P., A theoretical and experimental study of manganese oxides used as catalysts for VOCs emission reduction. Catal. Today 2005; 107:133-138.

9. Lou Y., Wang L., Zhao Z., Zhang Y., Lu G., Guo Y., Low-temperature CO oxidation over Co3O4-based catalysts: Significant promoting effect of $\mathrm{Bi} 2 \mathrm{O} 3$ on $\mathrm{Co}_{3} \mathrm{O}_{4}$ catalyst, Appl. Catal. B 2014; 146: 43- 49.

10. T. Garcia, S. Agouram, J.F. Sanchez-Royo, R. Murillo, A.M. Mastral, A. Aranda, I. Vazquez, A. Dejoz, B. Solsona, Deep oxidation of volatile organic compounds using ordered cobalt oxides prepared by a nanocasting route, Appl. Catal. A 2010; 386: 16-27

11. Hosseini A., Niaei A., Salari D., Nabavi S., Nanocrystalline AMn2O4 (A = Co, Ni, Cu) spinels for remediation of volatile organic compounds - synthesis, characterization and catalytic performance, Ceram. Int. 2012; 38: 1655-1661

12. Wyrwalski F., Lamonier J.F., Siffert S., Gengembre L., Aboukais A., Modified $\mathrm{Co}_{3} \mathrm{O}_{4} /$ ZrO2 catalysts for VOC emissions abatement, Catal. Today 2007;119: 332-337.

13. Konova P.,Stoyanova M., Naydenov A., Christoskova S., Mehandjiev D., Catalytic oxidation of VOCs and CO by ozone over alumina supported cobalt oxide, Appl. Catal. A 2006; 298: 109-114.

14. Todorova S., Kadinov G., Tenchev K., Caballero A., Holgado J., Catalysts for n-Hexane and $\mathrm{CO}$ Oxidation for $\mathrm{Co}_{3} \mathrm{O}_{4}+\mathrm{CeO}_{2} / \mathrm{SiO}_{2}$, Catal. Lett. 2009; 129: 149-155.

15. Tang C., Kuo C.C., Kuo M., Wang C.B., Chien H.B., Influence of pretreatment conditions on low-temperature carbon monoxide oxidation over $\mathrm{CeO}_{2} / \mathrm{Co}_{3} \mathrm{O}_{4}$ catalysts, Appl. Catal. 
A 2006; 309: 37-43.

16. Fierro J.L.G. (Ed.), Metal oxides.Chemistry and Applications, CRC Press, New York, (2006).

17. Aguilera D., Perez A., Molina R., Moreno S., Cu-Mn and Co-Mn catalysts synthesized from hydrotalcites and their use in the oxidation of VOCs, Appl. Catal. B 2011; 104: 144-150.

18. Perez A., Montes M., Molina R., Moreno S., Cooperative effect of Ce and Pr in the catalytic combustion of ethanol in mixed $\mathrm{Cu} / \mathrm{CoMgAl}$ oxides obtained from hydrotalcites, Appl. Catal. A 2011; 408: 96-104.

19. Castaño, M., Molina, R., Moreno, S., Mn-Co-Al-Mg mixed oxides by auto-combustion method and their use as catalysts in the total oxidation of toluene, J. Mol. Catal. A 2013; 370: $167-174$.

20. Li J., Liang, S., Hao J., Catalytic performance of manganese cobalt oxides on methane combustion at low temperature, Appl. Catal. B 2009: 307-312.

21. Picasso G., Cruz R., Sun Kou R., Preparation by co-precipitation of Ce-Mn based catalysts for combustion of n-hexane, Mat. Res. Bull. 2015; 70: 621-632.

22. Puértolas B., Smith A., Vázquez I., Dejoz A., Moragues A., Garcia T., Solsona B., The different catalytic behavior in the propane total oxidation of cobalt and manganese oxides prepared by a wet combustion procedure, Chem. Eng. J. 2013; 229: 547-5585160 .

23. Todoroba, S., Kolev H. Holgado J.P., Kadinov G., Bonev Ch., Pereñiguez R., Caballero A., Complete n-hexane oxidation over supported Mn-Co catalysts. Appl. Catal. B 2010; 94: 46-54.

24. Todoroba, S., Naydenov A., Kolev, H., Holgado, J.P., Ivanov, G., Kadinov G., Caballero A., Mechanism of complete n-hexane oxidation on silica supported cobalt and manganese catalysts. Appl. Catal. A 2012; 413-414: 43-51. 